\title{
Tailoring Supramolecular Peptide-Poly(ethylene glycol) Hydrogels by Coiled Coil Self-Assembly and Self-Sorting
}

Staffan Dånmark ${ }^{\ddagger}$, Christopher Aronsson, and Daniel Aili*

Division of Molecular Physics, Department of Physics, Chemistry and Biology, Linköping University, 581 83, Linköping, Sweden

$\$$ These authors contributed equally to this work

*daniel.aili@liu.se

\section{Supporting Information}

\section{Table of contents}

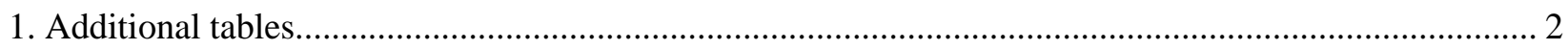

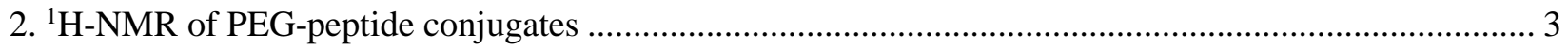

3. CD spectra and thermal stability curves of individual and mixed PEG-peptide conjugates ................... 4

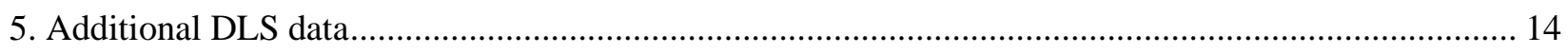

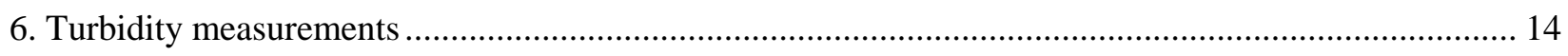

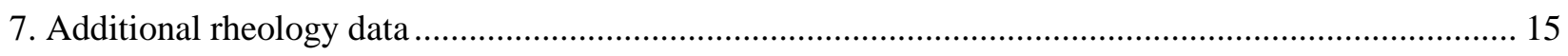

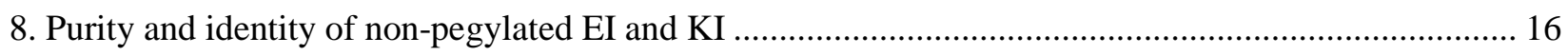




\section{Additional tables}

Table S1. Melting temperatures ( $\left.T_{m}\right)$ and $K_{d}$ of non-pegylated peptides, adapted from Aronsson et al. ${ }^{1}$

\begin{tabular}{|c|c|c|}
\hline Non-pegylated peptides & $\mathbf{T}_{\mathbf{m}}\left({ }^{\circ} \mathbf{C}\right)$ & $\mathbf{K}_{\mathbf{d}}$ at $\mathbf{2 0}^{\circ} \mathbf{C}(\mathbf{M})$ \\
\hline $\mathrm{EI}$ & $<20$ & --- \\
\hline $\mathrm{EV}$ & $<5$ & --- \\
\hline $\mathrm{KI}$ & $<20$ & --- \\
\hline $\mathrm{KV}$ & $<5$ & --- \\
\hline $\mathrm{EI} / \mathrm{KI}$ & $89^{*}$ & $<1.0 \times 10^{-10}$ \\
\hline $\mathrm{EV} / \mathrm{KI}$ & $63^{*}$ & $8.5 \pm 7.1 \times 10^{-8}$ \\
\hline $\mathrm{EI} / \mathrm{KV}$ & $66^{*}$ & $7.2 \pm 4.7 \times 10^{-8}$ \\
\hline $\mathrm{EV} / \mathrm{KV}$ & $32^{*}$ & $1.4 \pm 1.5 \times 10^{-6}$ \\
\hline
\end{tabular}

* Estimated $\mathrm{T}_{\mathrm{m}}$ at $10 \mu \mathrm{M}$ total peptide concentration

Table S2. MRE 222 and melting temperatures $\left(\mathrm{T}_{\mathrm{m}}\right)$ of individual PEG-conjugates at different $\mathrm{pH}$.

\begin{tabular}{|c|c|c|c|c|c|c|c|c|}
\hline & \multicolumn{2}{|c|}{$\mathrm{pEV}_{4}$} & \multicolumn{2}{|c|}{$\mathrm{pKV}_{4}$} & \multicolumn{2}{|c|}{$\mathbf{p E I}_{4}$} & \multicolumn{2}{|c|}{$\mathrm{pKI}_{4}$} \\
\hline pH & $\begin{array}{c}\text { MRE }_{222} * \\
20^{\circ} \mathrm{C}\end{array}$ & $\mathbf{T}_{\mathrm{m}}\left({ }^{\circ} \mathbf{C}\right)$ & $\begin{array}{c}{\text { MRE } 222^{*}} \\
20^{\circ} \mathrm{C}\end{array}$ & $\mathbf{T}_{\mathrm{m}}\left({ }^{\circ} \mathbf{C}\right)$ & $\begin{array}{c}\text { MRE }_{222} * \\
20^{\circ} \mathrm{C}\end{array}$ & $\mathbf{T}_{\mathrm{m}}\left({ }^{\circ} \mathbf{C}\right)$ & $\begin{array}{c}\text { MRE } 222^{*} \\
20^{\circ} \mathrm{C}\end{array}$ & $\mathbf{T}_{\mathbf{m}}\left({ }^{\circ} \mathbf{C}\right)$ \\
\hline 4 & -20.3 & $58.7 \pm 1.7$ & -5.0 & $<5$ & -35.3 & $>90$ & -22.1 & $28.5 \pm 1.6$ \\
\hline 5 & -6.7 & $<5$ & -3.1 & $<5$ & -33.0 & $75.4 \pm 2.2$ & -20.2 & $30.3 \pm 0.8$ \\
\hline 6 & -3.4 & $<5$ & -3.8 & $<5$ & -30.5 & $36.9 \pm 1.2$ & -26.2 & $31.8 \pm 0.5$ \\
\hline 7 & -3.5 & $<5$ & -2.5 & $<5$ & -8.5 & $<20$ & -27.5 & $42.7 \pm 0.2$ \\
\hline 8 & -3.0 & $<5$ & -3.4 & $<5$ & -10.5 & $<20$ & -32.1 & $53.3 \pm 0.6$ \\
\hline 9 & -3.4 & $<5$ & -3.5 & $<5$ & -9.6 & $<20$ & -30.7 & $75.4 \pm 4.2$ \\
\hline 10 & -2.3 & $<5$ & -3.6 & $<5$ & -9.4 & $<20$ & -26.5 & $>90$ \\
\hline
\end{tabular}

* Expressed as mean residue ellipticity, $\left(10^{3} \mathrm{deg}_{\mathrm{cm}} \mathrm{dmol} \mathrm{res}^{-1}\right)$ 


\section{2. ${ }^{1} \mathrm{H}-\mathrm{NMR}$ of PEG-peptide conjugates}
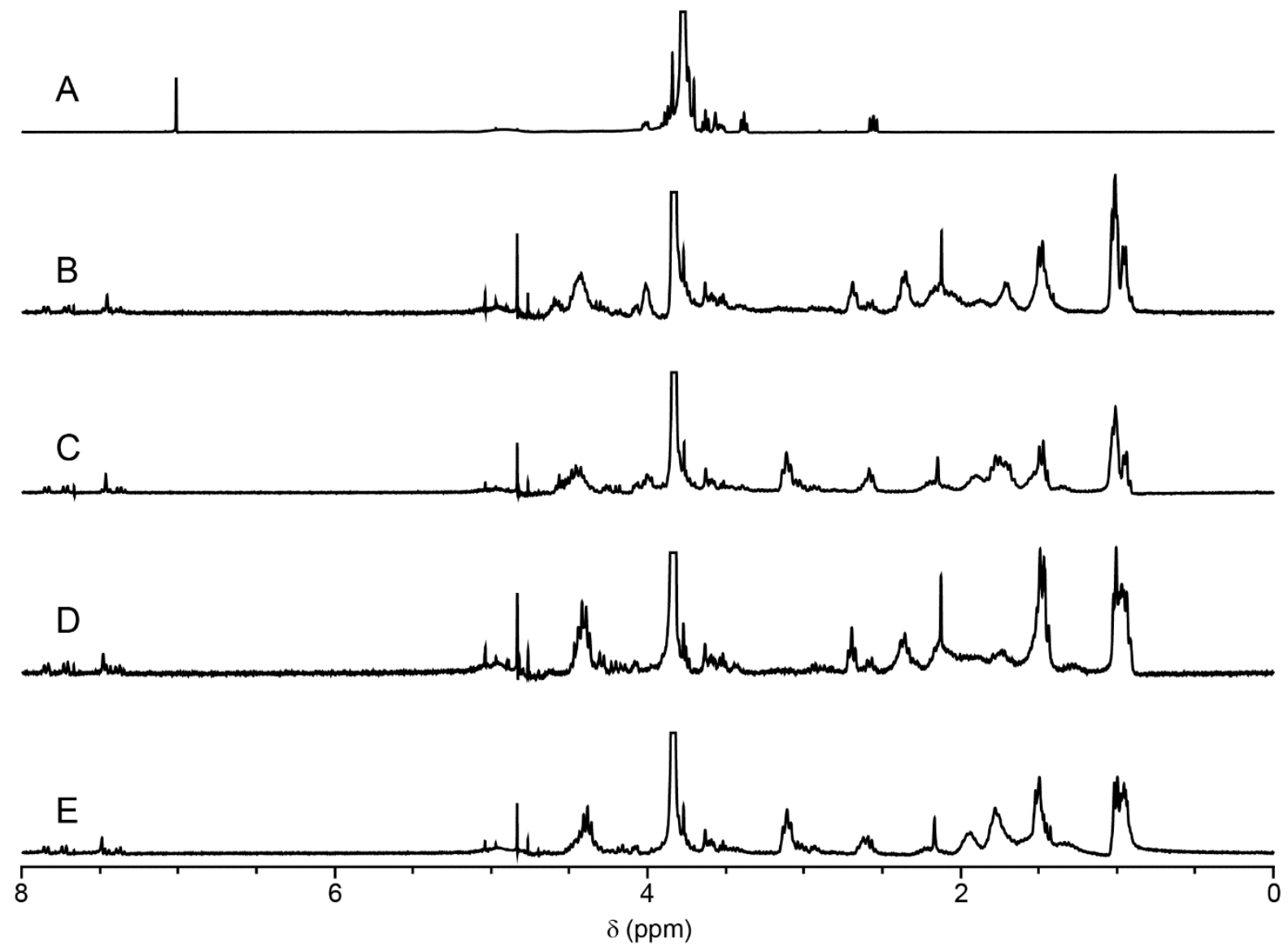

Figure S1. ${ }^{1} \mathrm{H}-\mathrm{NMR}$ of (A) maleimide functionalized 4-armed polyethylene glycol, and subsequently with conjugated peptides (B) $\mathrm{pEV}_{4}$, (C) $\mathrm{pKV}_{4}$, (D) $\mathrm{pEI}_{4}$ and (E) $\mathrm{pKI}_{4}$. Note the disappearing maleimide group (300 MHz, $\left.\mathrm{D}_{2} \mathrm{O}, \delta\right): 6.86(\mathrm{~s}, 2 \mathrm{H}, \mathrm{C}-\mathrm{H})$ and introduction of new peaks from peptide residues (e.g. $\operatorname{Trp}\left(300 \mathrm{MHz}, \mathrm{D}_{2} \mathrm{O}, \delta\right): 7.3(\mathrm{~m}$, $4 \mathrm{H}, \mathrm{Ar}-\mathrm{H})$. 


\section{CD spectra and thermal stability curves of individual and mixed PEG-peptide conjugates}
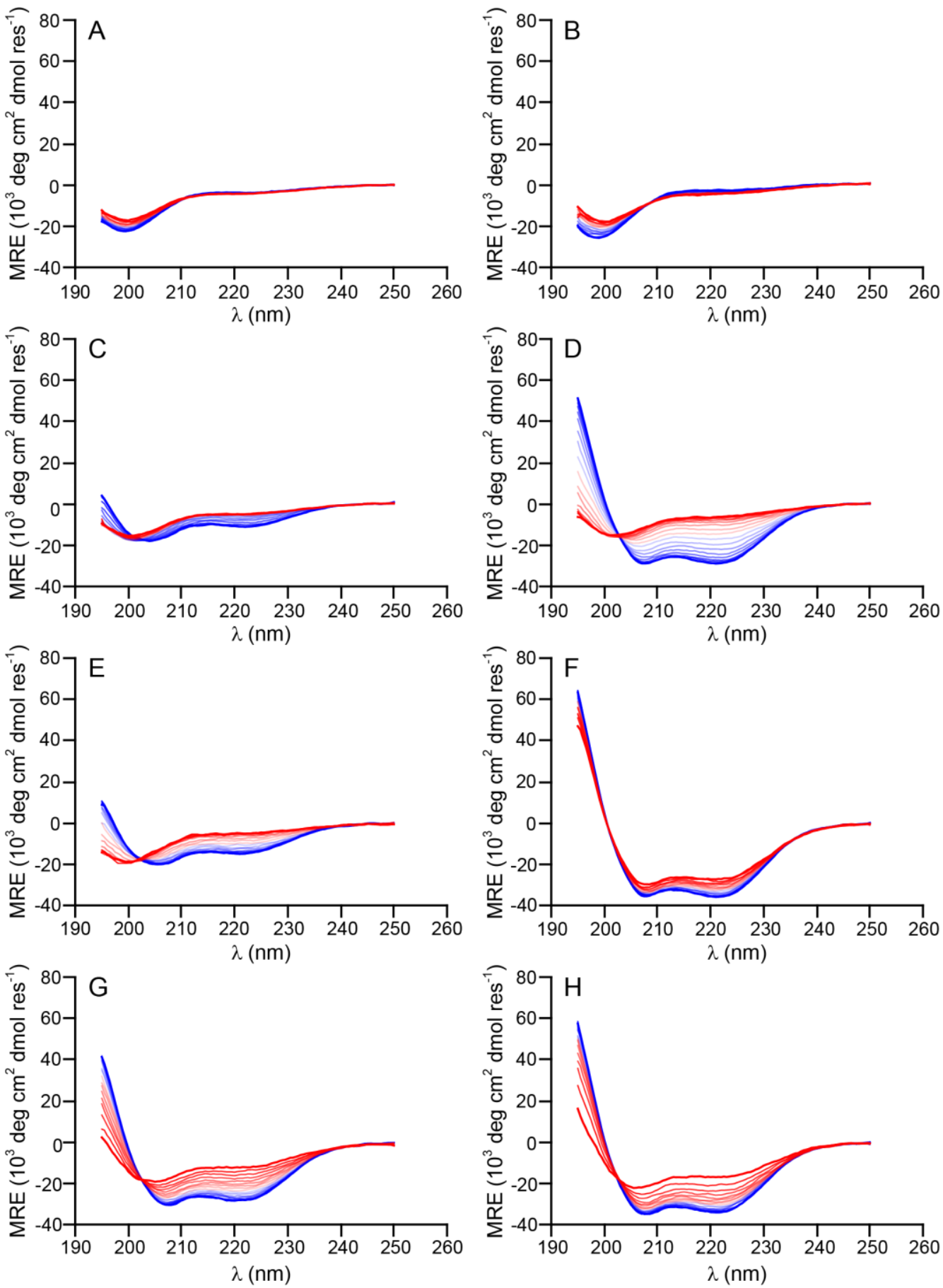

Figure S2. Complete CD spectra of individual and mixed PEG-peptide conjugates at different temperatures and at $\mathrm{pH}$ 7. Key: "Blue to red" gradient is $5-90{ }^{\circ} \mathrm{C}$ in steps of $5{ }^{\circ} \mathrm{C}$. (A) $\mathrm{pEV}_{4}$, (B) $\mathrm{pKV}_{4},(\mathrm{C}) \mathrm{pEI}_{4},(\mathrm{D}) \mathrm{pKI}_{4},(\mathrm{E})$ pEV4/pKV 4 , (F) $\mathrm{pEI}_{4} / \mathrm{pKI}_{4},(\mathrm{G}) \mathrm{pEV}_{4} / \mathrm{pKI}_{4}$ and $(\mathrm{H}) \mathrm{pEI}_{4} / \mathrm{pKV}_{4}$. 

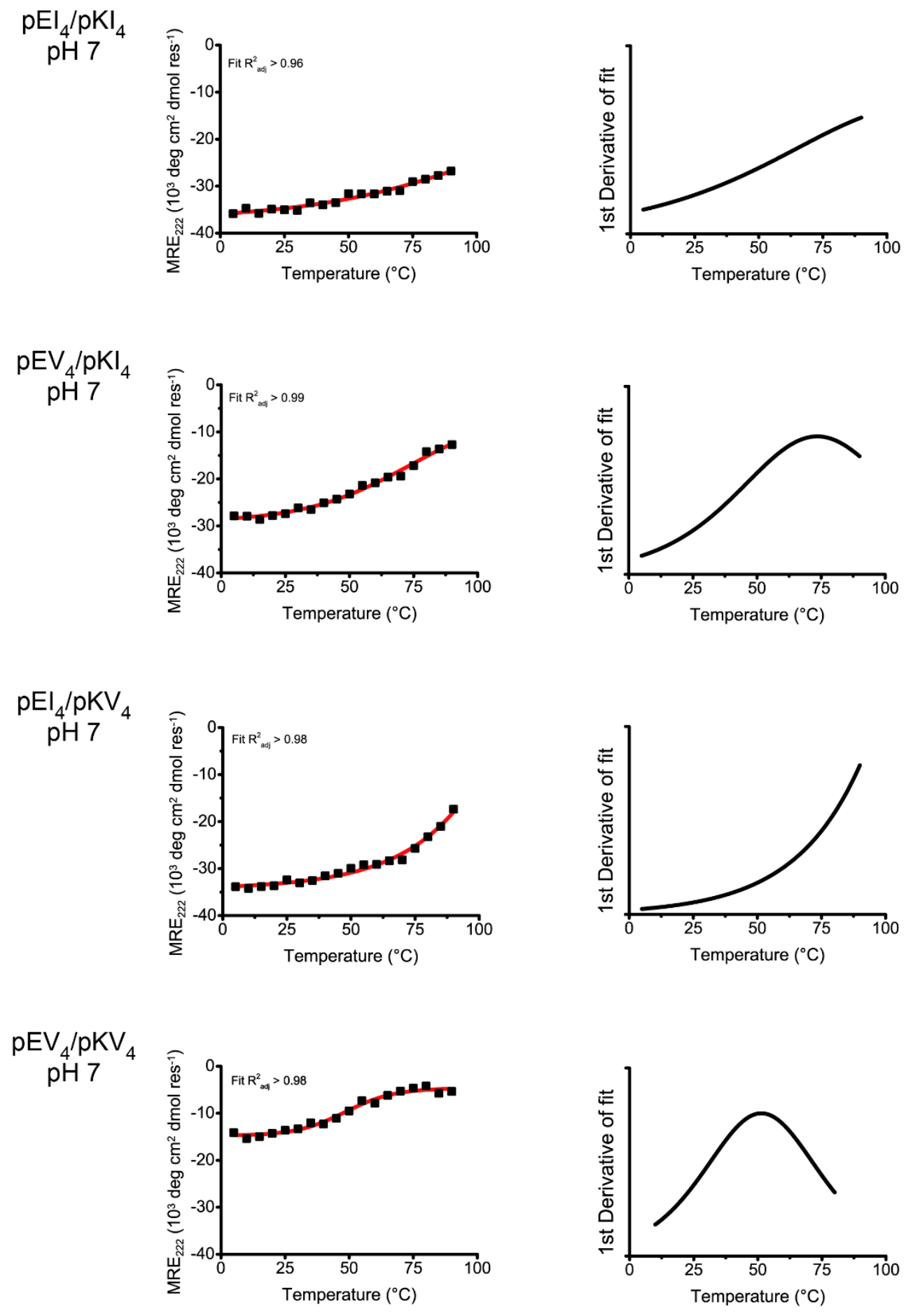
$\mathrm{pEI}_{4}, \mathrm{pH} 4$

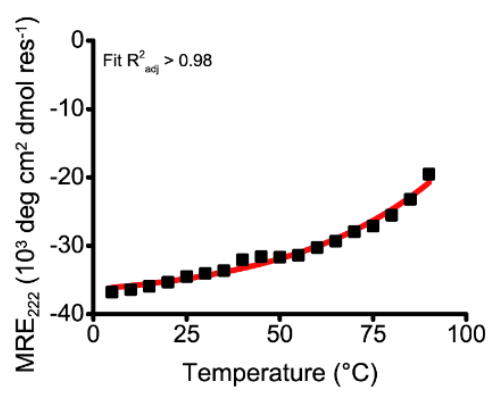

$\mathrm{pEI}_{4}, \mathrm{pH} 5$

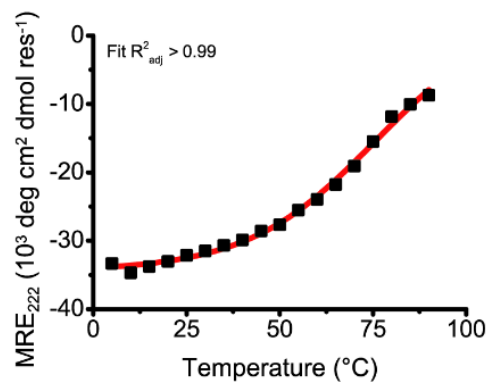

$\mathrm{pEI}_{4}, \mathrm{pH} 6$

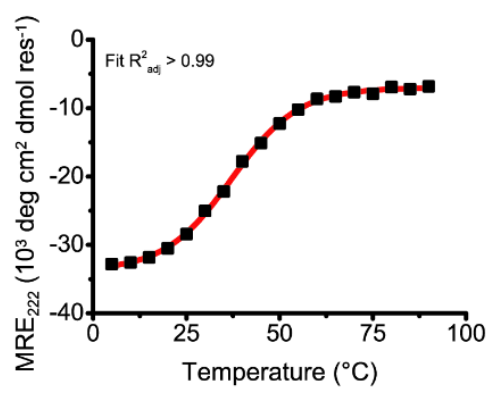

$\mathrm{pEI}_{4}, \mathrm{pH} 7$

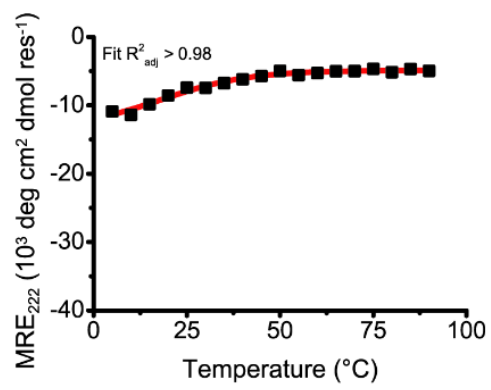

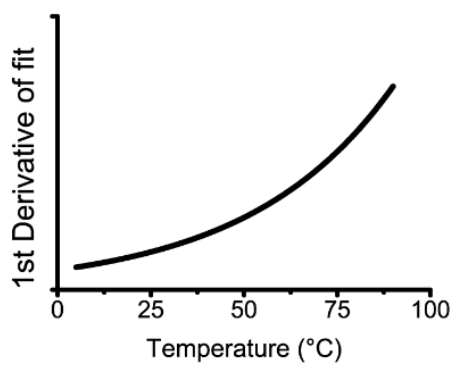
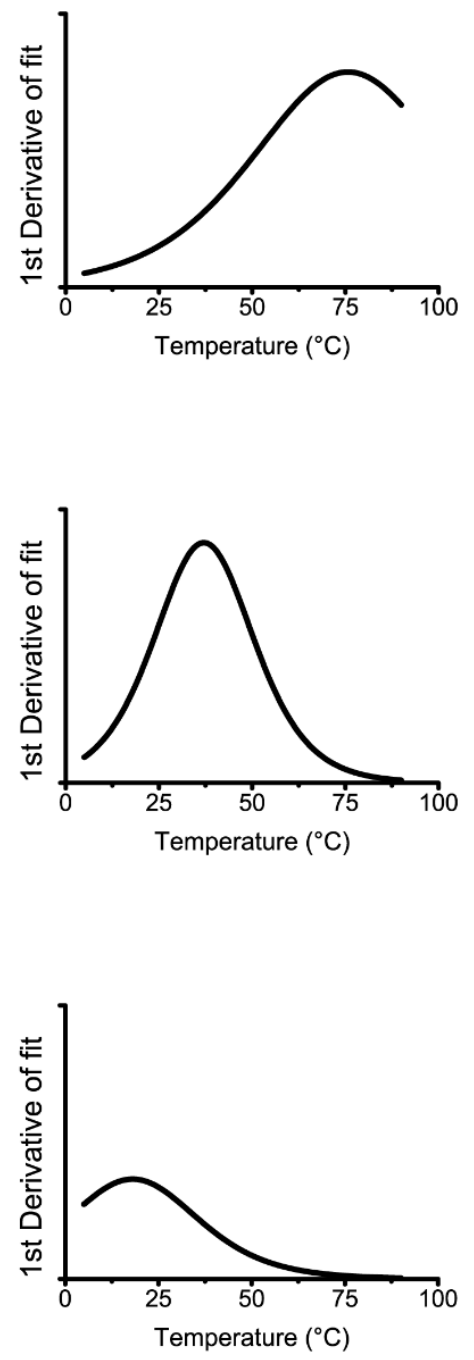

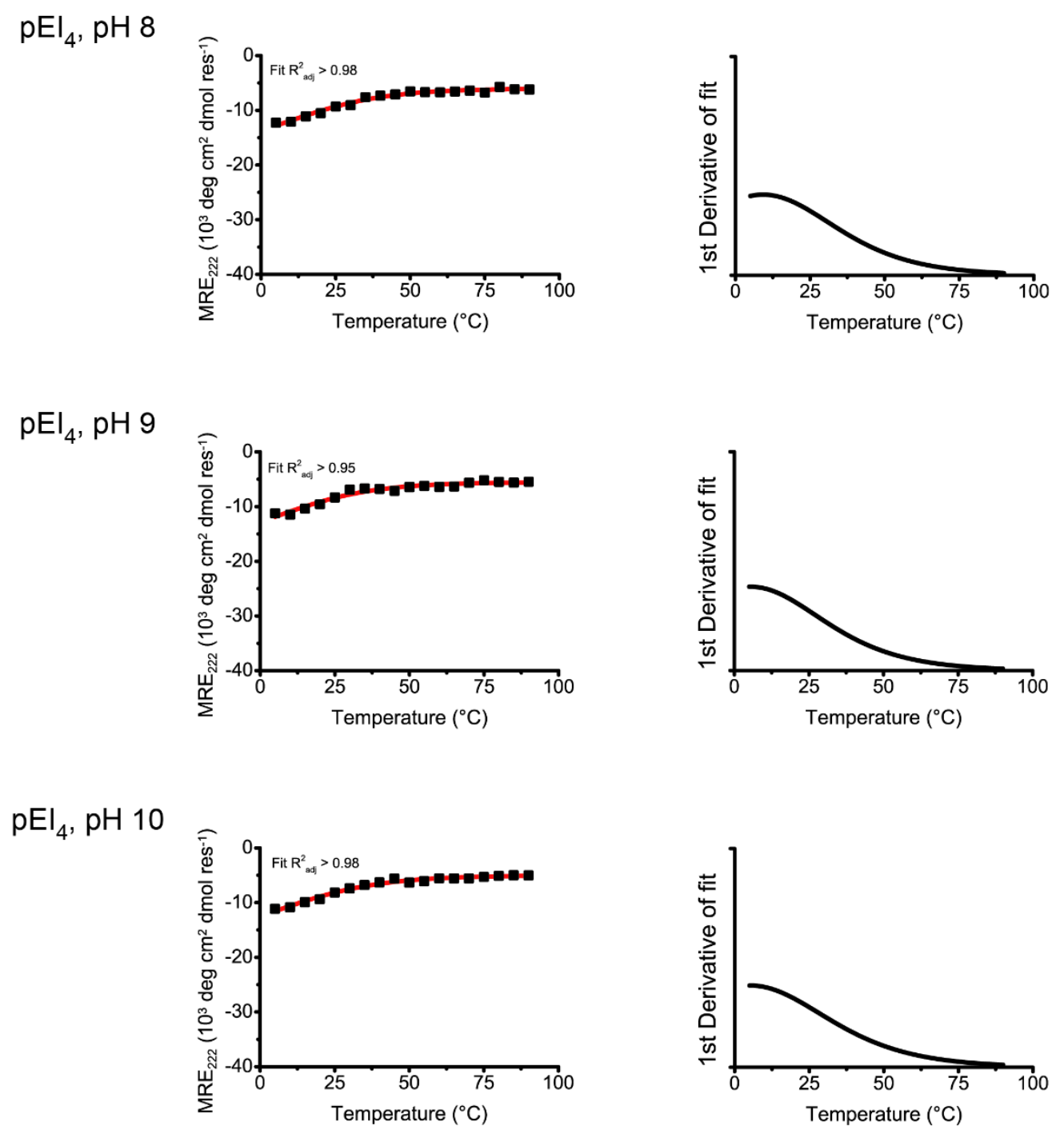

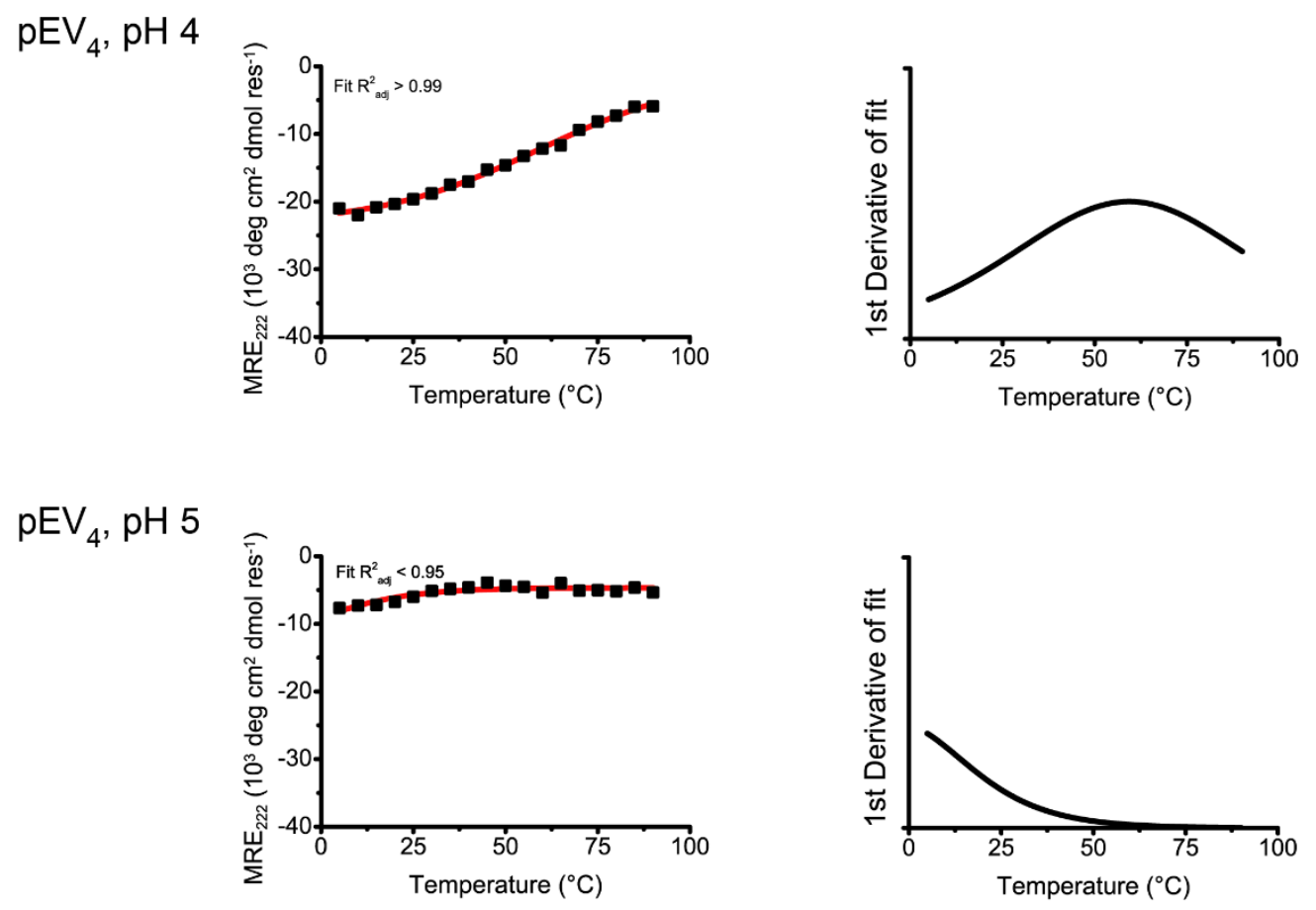

$\mathrm{pEV}_{4}, \mathrm{pH} 6$
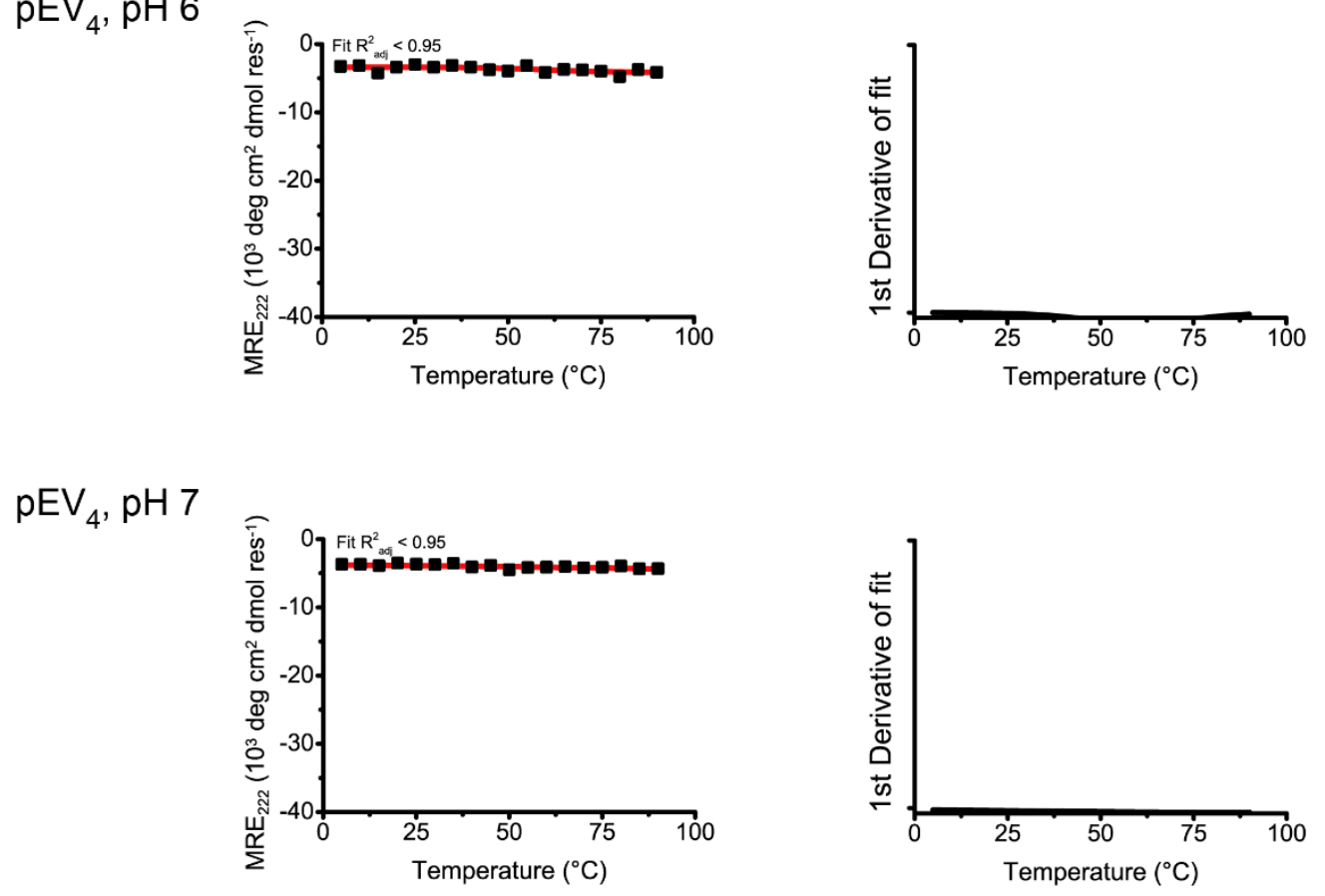

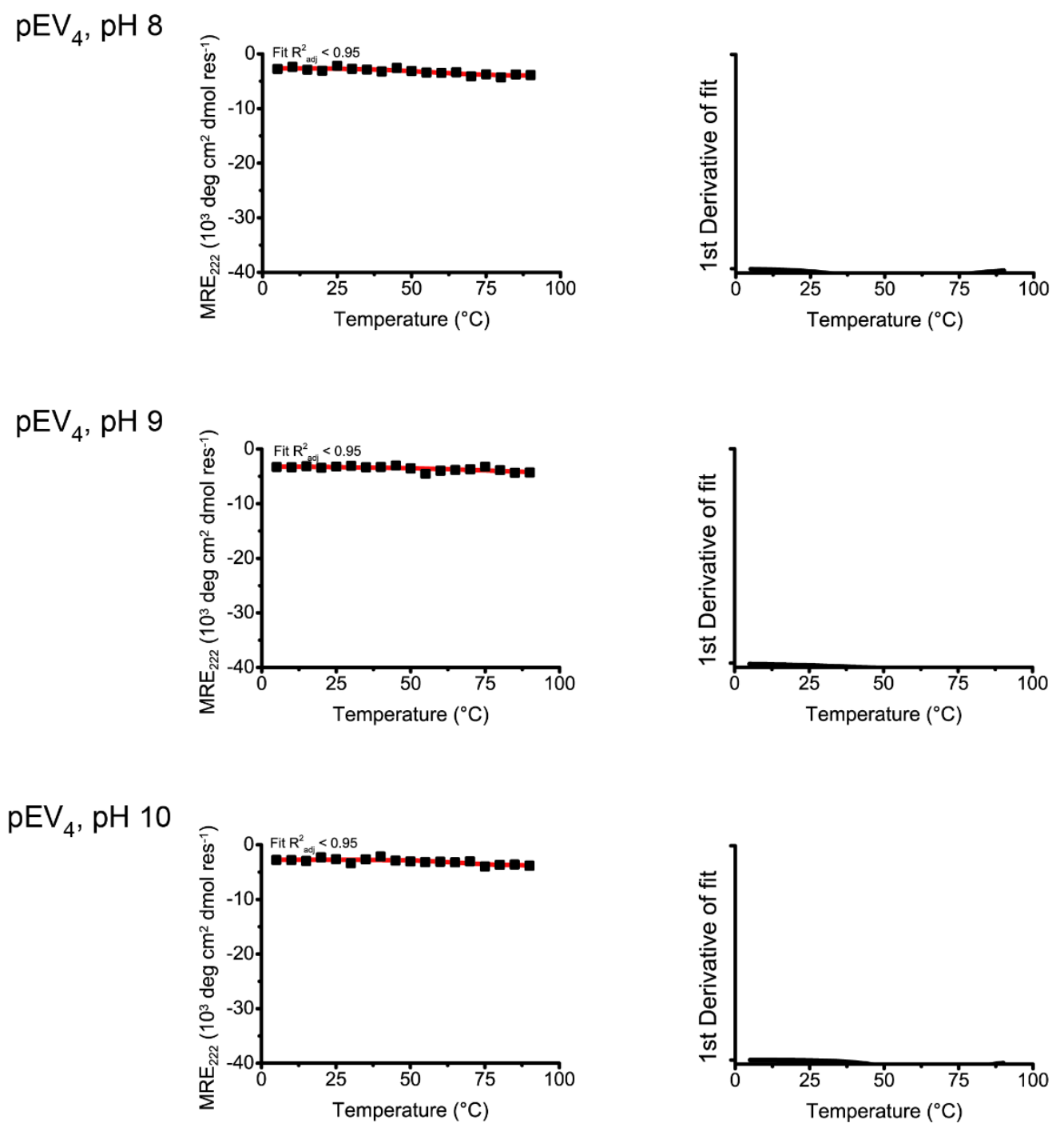

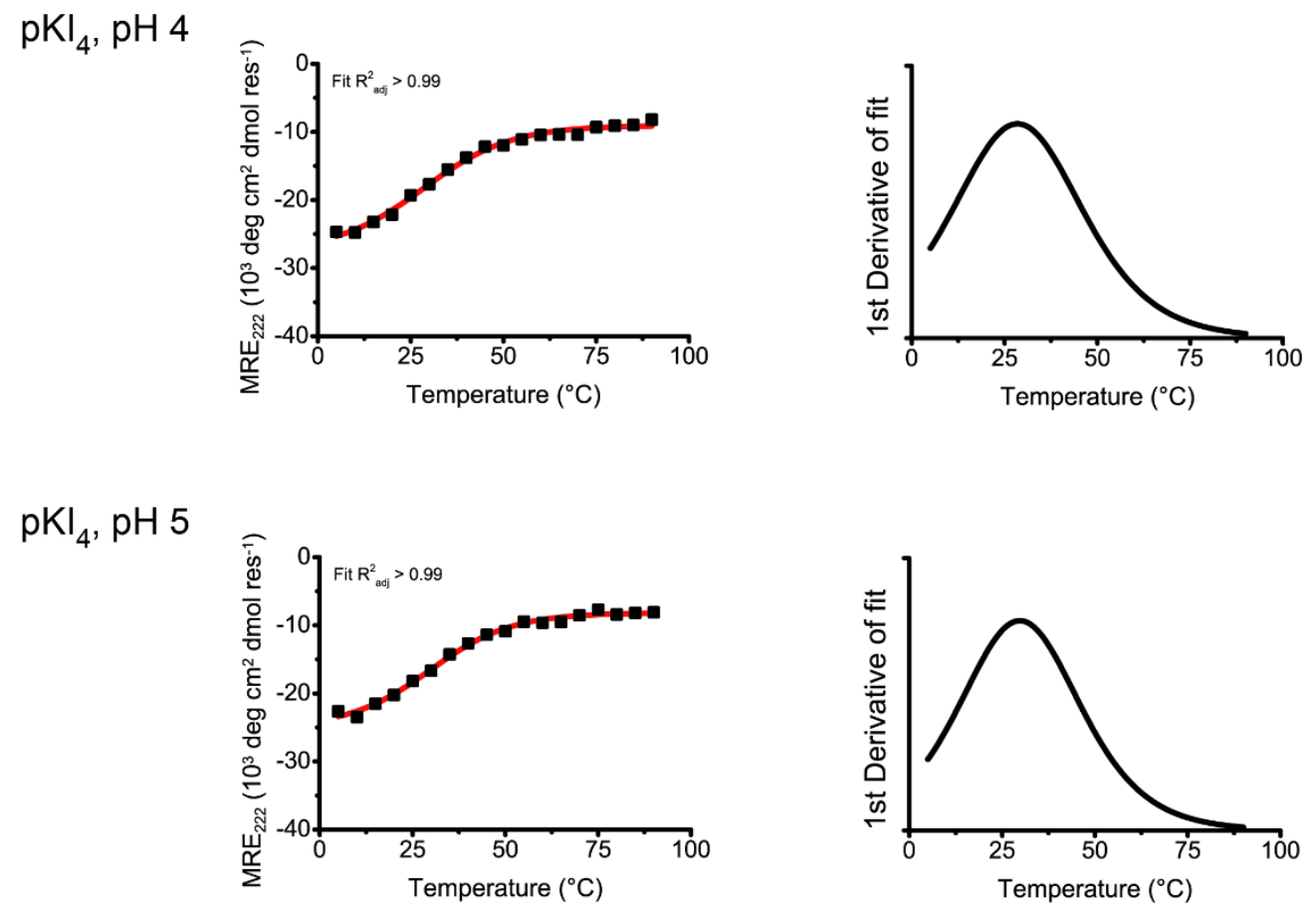

$\mathrm{pKI}_{4}, \mathrm{pH} 6$
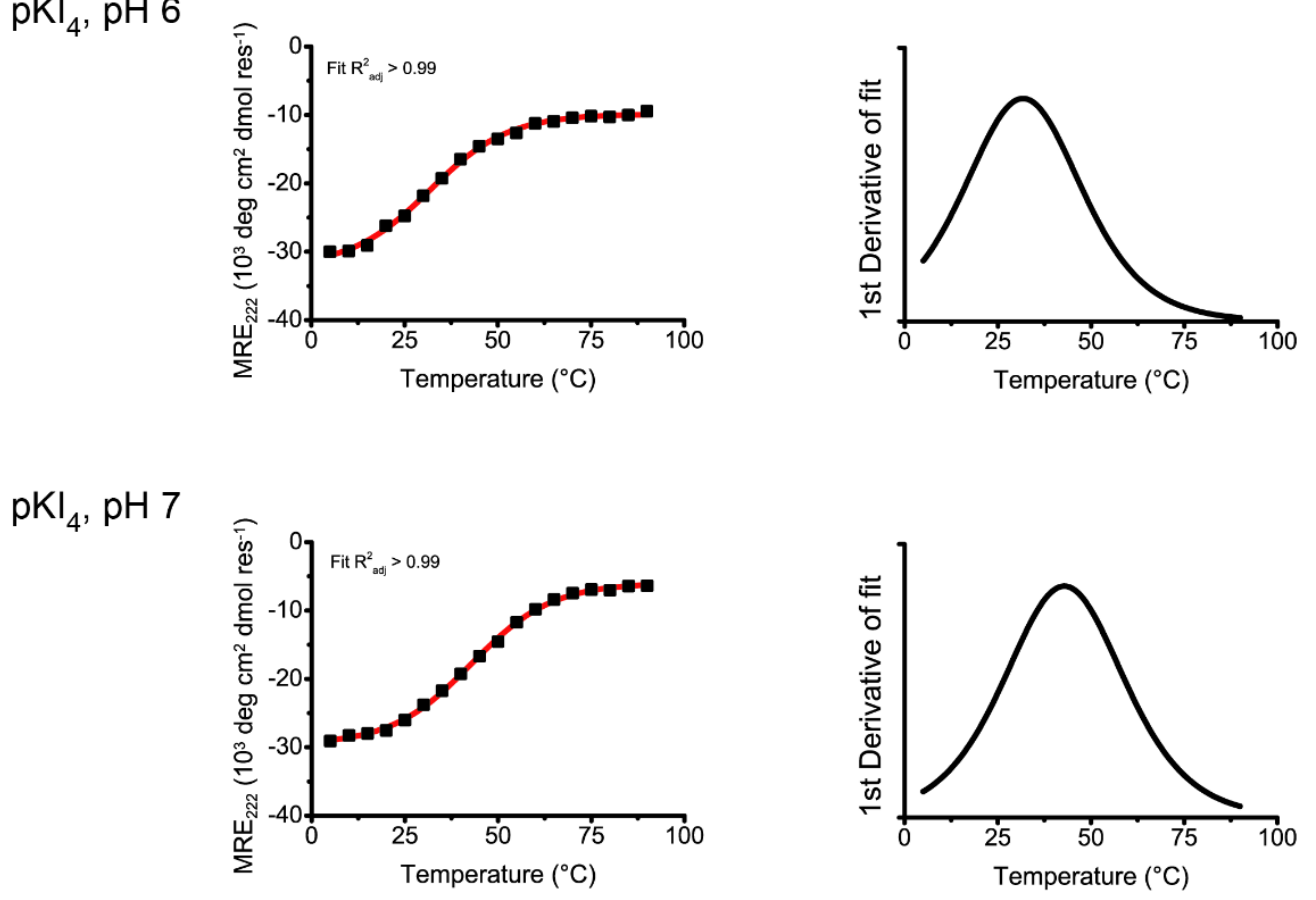

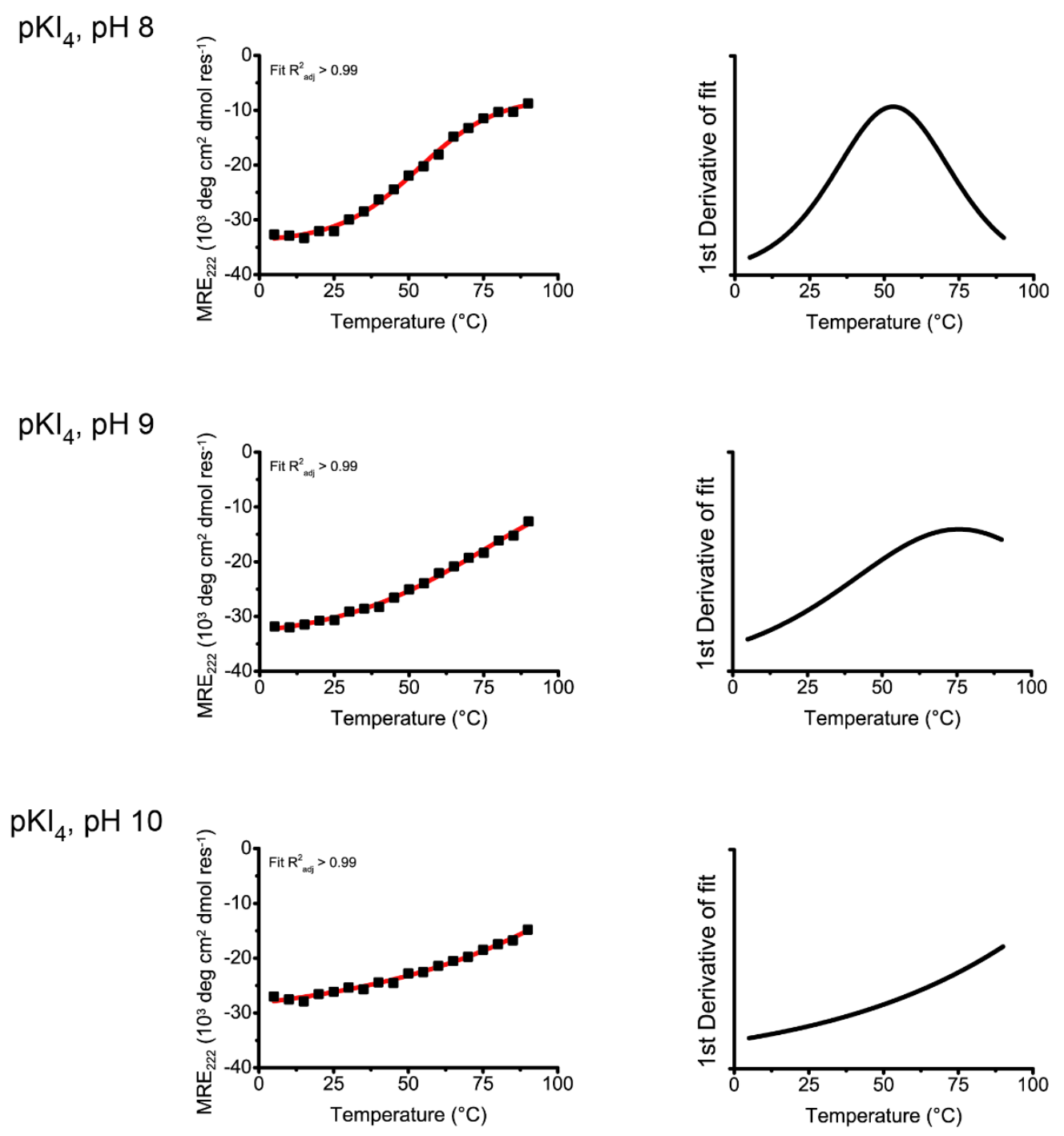

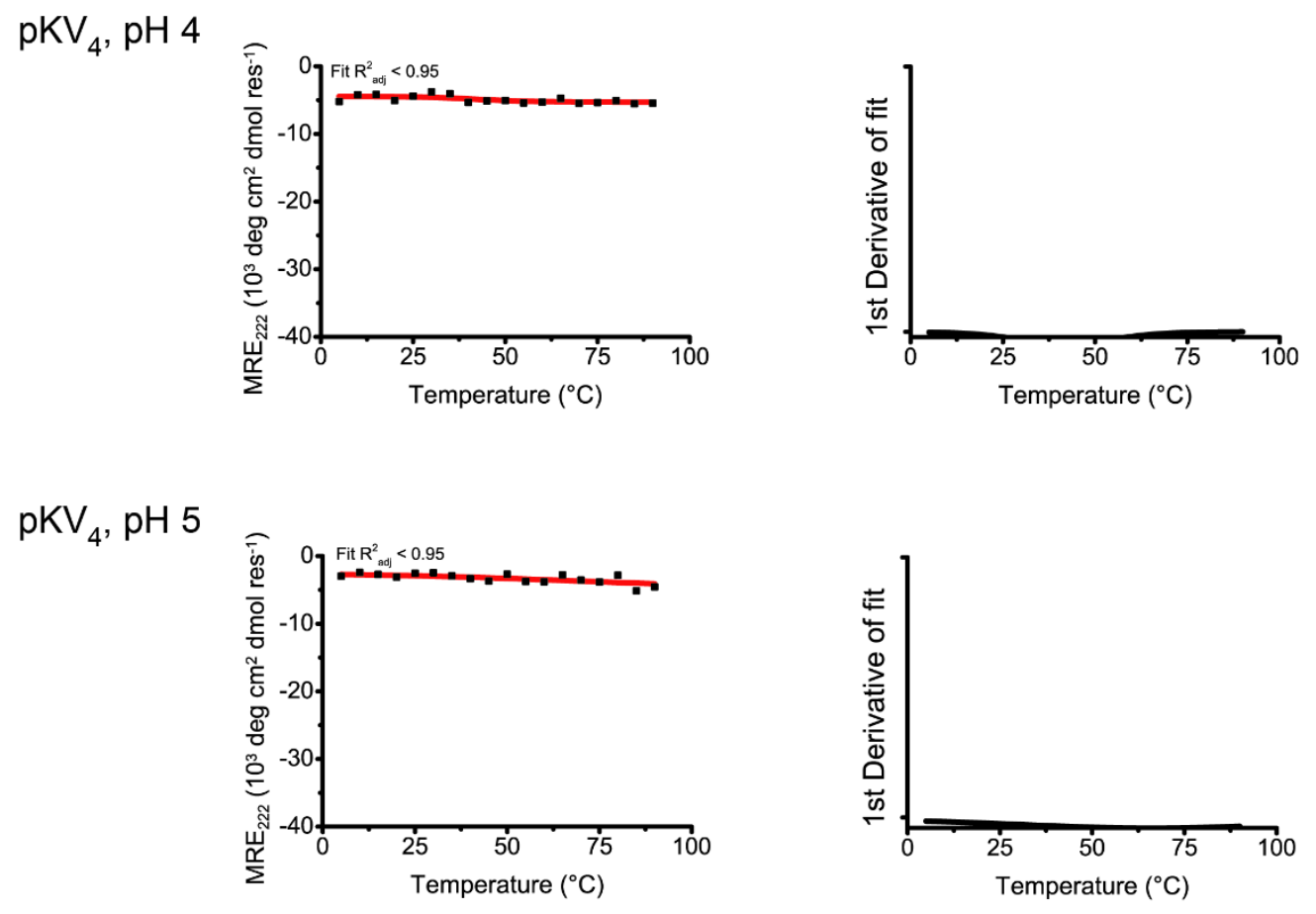

$\mathrm{pKV}_{4}, \mathrm{pH} 6$
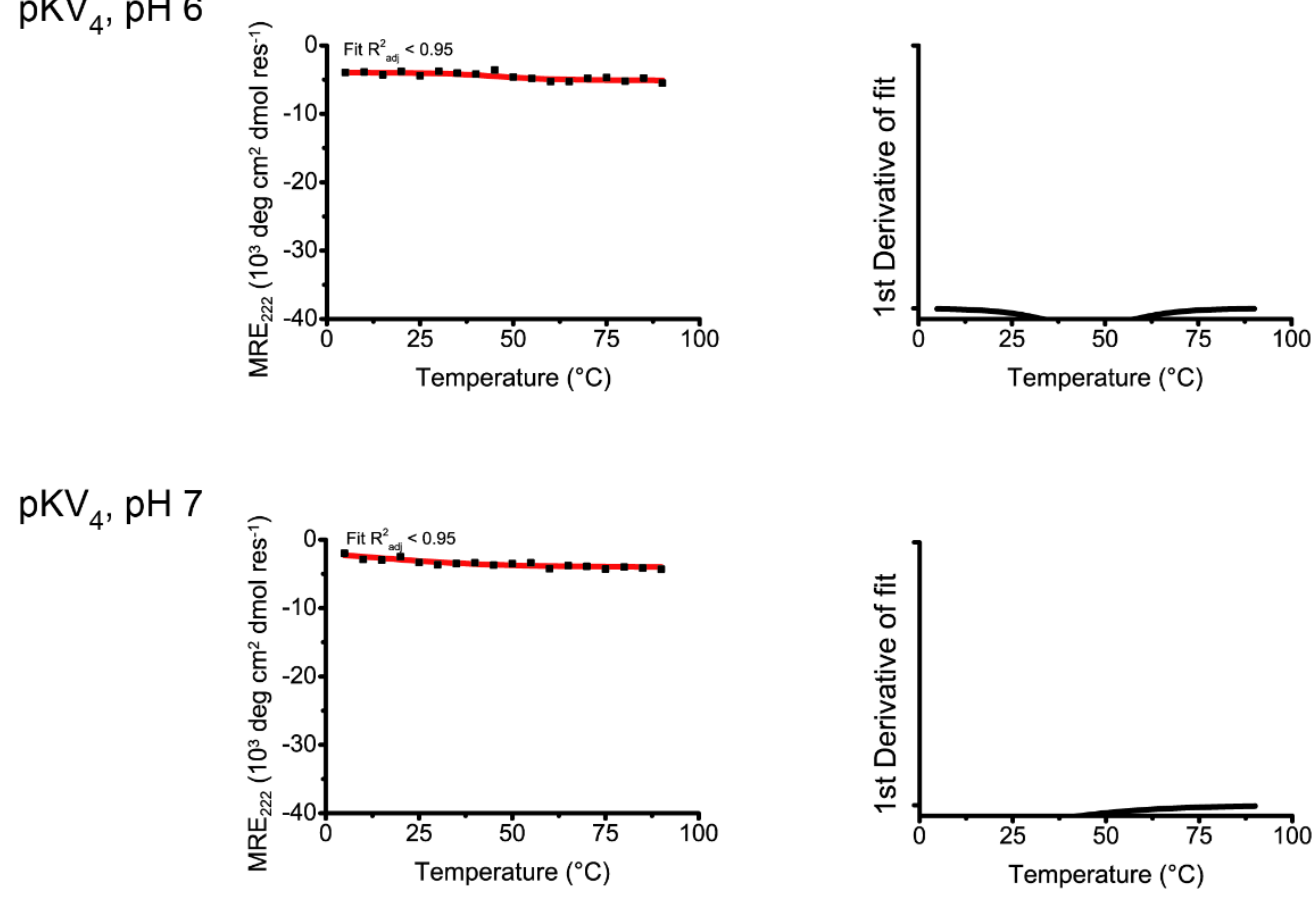

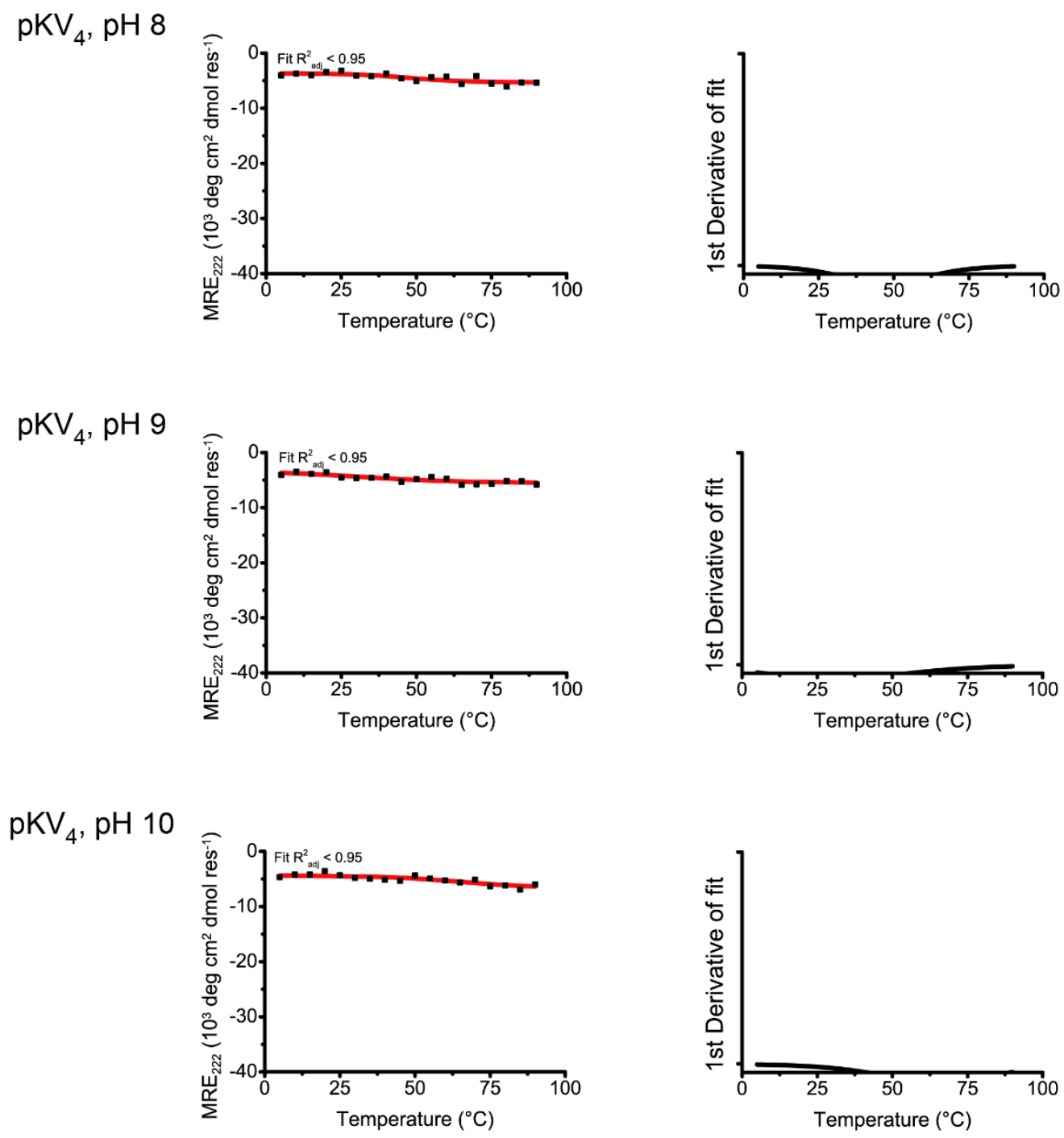


\section{Additional DLS data}
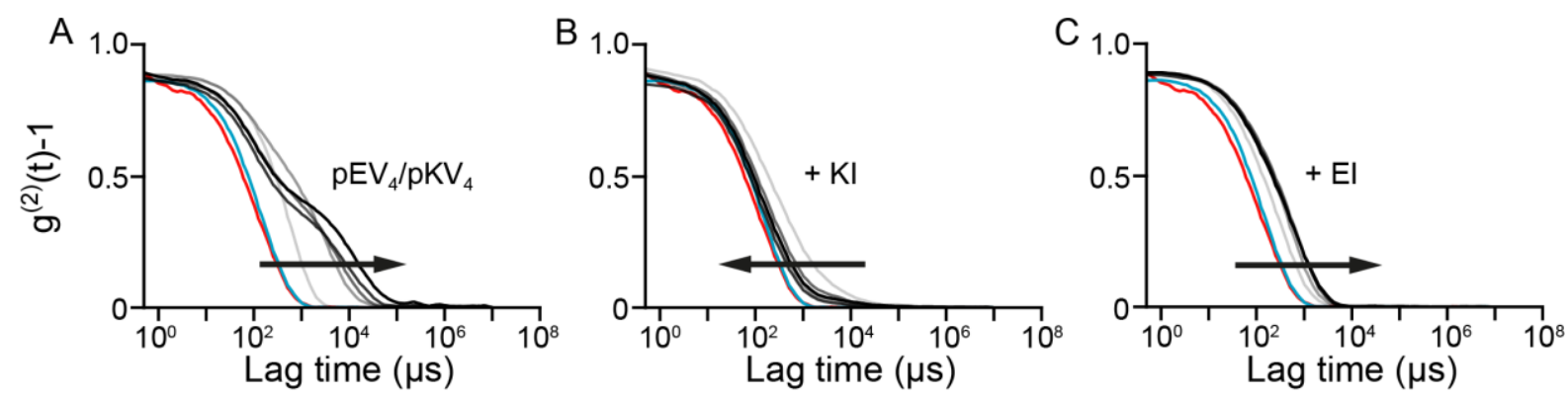

Figure S3. (A) Mixing $\mathrm{pEV}_{4}$ and $\mathrm{pKV}_{4}$ creates the PEG-peptide network $\mathrm{pEV}_{4} / \mathrm{pKV}_{4}$. (B) Adding non-pegylated KI will disrupt the PEG network, caused by formation of $\mathrm{pEV}_{4} / 4 \mathrm{KI}$. (C) Subsequential addition of non-pegylated EI will reform the PEG network, since EI/KI will form and as a consequence $\mathrm{pEV}_{4} / \mathrm{pKV}_{4}$ will be able to form once again.

\section{Turbidity measurements}

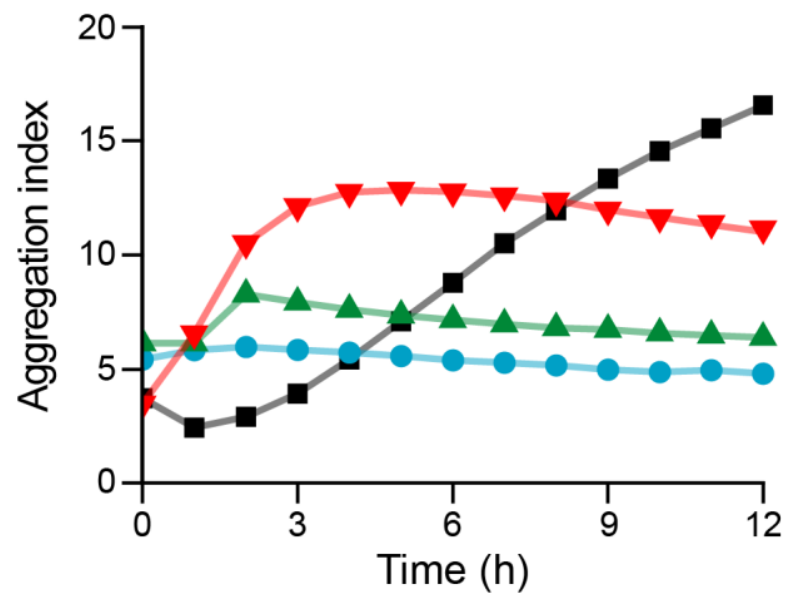

Figure S4. Turbidity measurement, expressed as aggregation index, over time for mixtures of PEG-peptide conjugates. Key: black, squares $=\mathrm{pEI}_{4} / \mathrm{pKI}_{4}$; red, upside down triangle $=\mathrm{pEI}_{4} / \mathrm{pKV}_{4}$; green, triangle $=\mathrm{pEV}_{4} / \mathrm{pKI}_{4}$; blue, circle $=\mathrm{pEV}_{4} / \mathrm{pKV}_{4}$. 


\section{Additional rheology data}
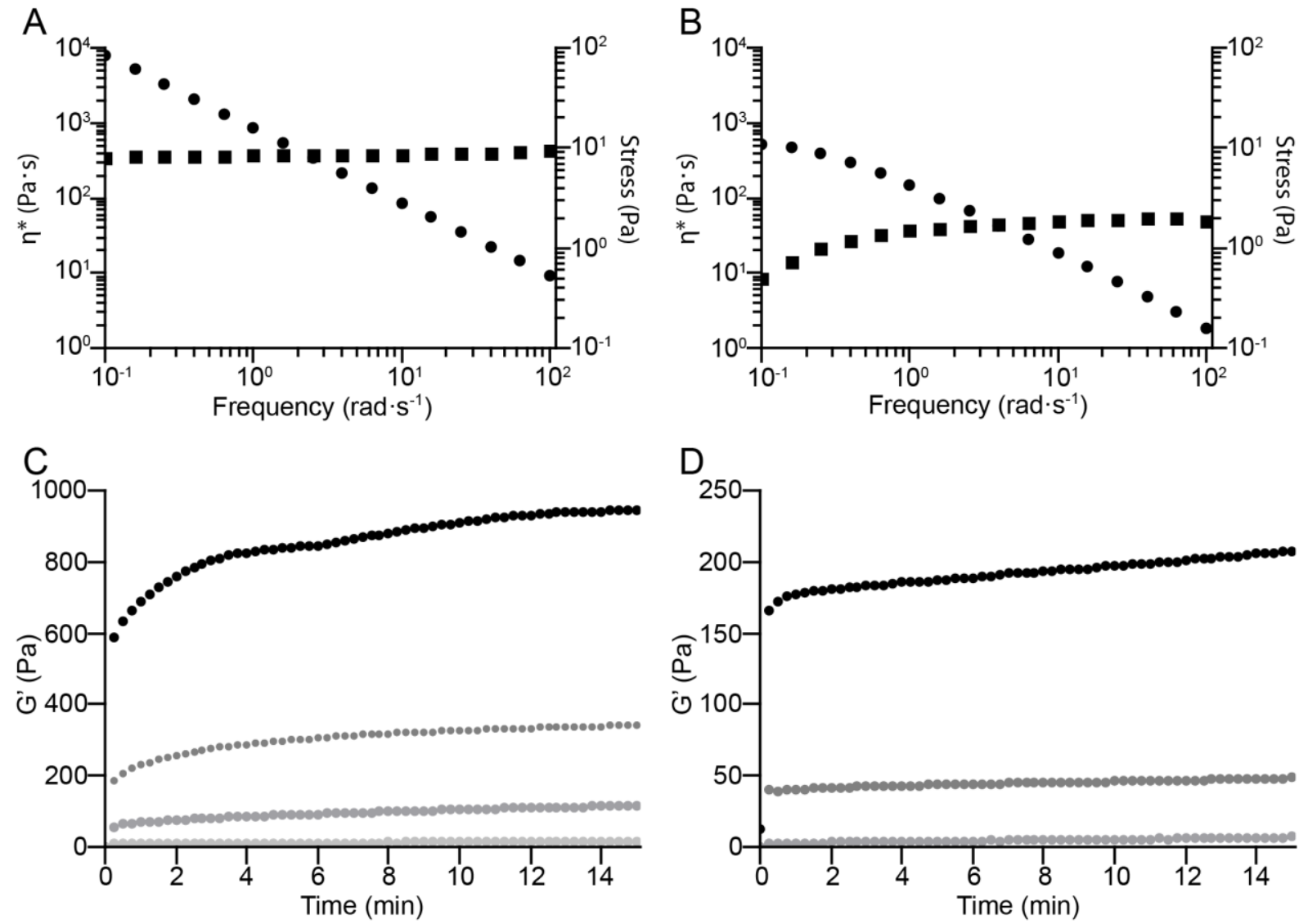

Figure S5. Additional rheology data for gels of $\mathrm{pEI}_{4} / \mathrm{pKI}_{4}$ and $\mathrm{pEI}_{4} / \mathrm{pKV}_{4}$. (A) and (B) show frequency sweep data showing the shear thinning behavior for (A) $\mathrm{pEI}_{4} / \mathrm{pKI}_{4}$ and (B) $\mathrm{pEI}_{4} / \mathrm{pKV}_{4}$ respectively. (C) and (D) shows restoration of gel strengths as a function of time after mechanical disruption of (C) $\mathrm{pEI}_{4} / \mathrm{pKI}_{4}$ and (D) $\mathrm{pEI} 4 / \mathrm{pKV}_{4}$ gels respectively. Key: $(A-B)$ Viscosity $\left(\eta^{*}\right)=$ circles and stress $=$ squares of $2.5 \%(w / v)$ gels. $(C-D) 2.5 \%(w / v)$ (black) to $1 \%(\mathrm{w} / \mathrm{v})$ (light grey) in steps of $0.5 \%$. 


\section{Purity and identity of non-pegylated EI and KI}

Non-pegylated EI

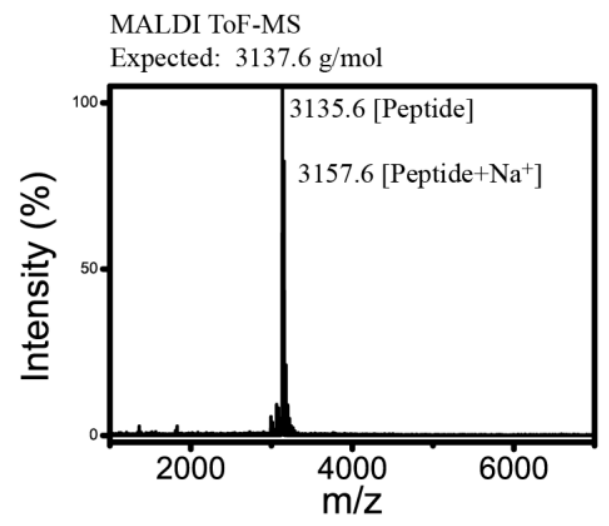

Non-pegylated KI

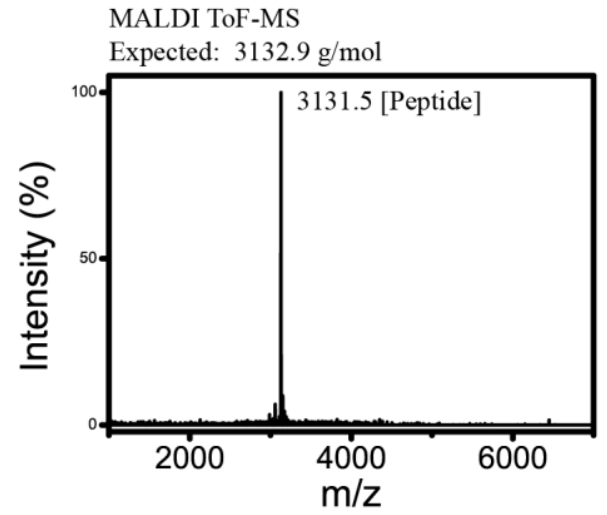

HPLC

Gradient 10 - $80 \%$ buffer D over $20 \mathrm{~min}$

Liquid phase:C $=90 \% 20 \mathrm{mM}$ TEAA-buffer, $10 \%$ Acetonitrile $\mathrm{D}=10 \% 20 \mathrm{mM}$ TEAA-buffer, $90 \%$ Acetonitrile Detected@UV=229 nm

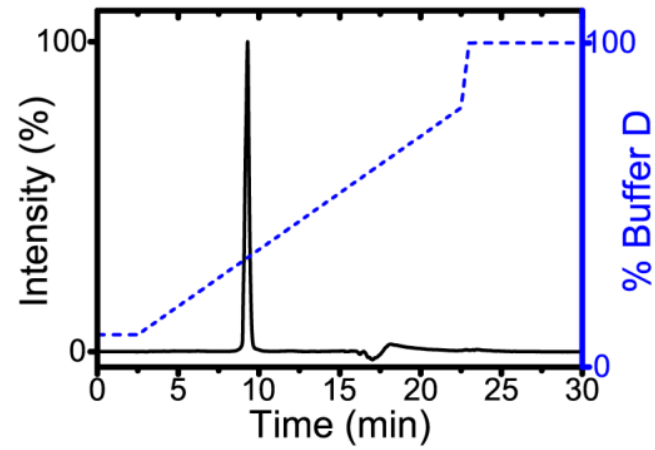

HPLC

Gradient: 10 - $80 \%$ buffer B over $20 \mathrm{~min}$

Liquid phase:A = $90 \%$ Water, $9.9 \%$ Acetonitrile, $0.1 \%$ TFA $\mathrm{B}=9.9 \%$ Water, $90 \%$ Acetonitrile, $0.1 \%$ TFA

Detected@UV=229nm

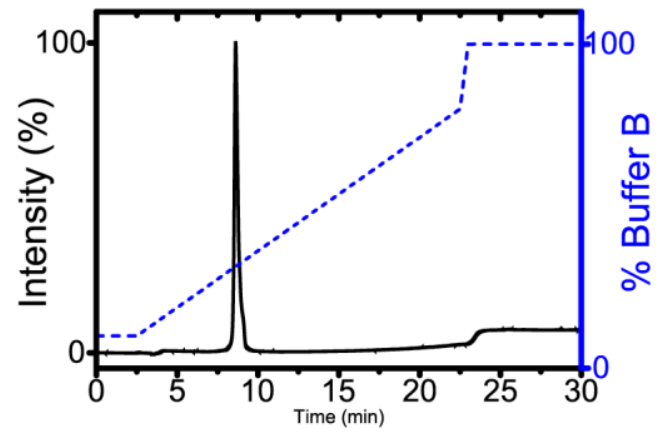

\section{References}

(1) Aronsson, C.; Dånmark, S.; Zhou, F.; Öberg, P.; Enander, K.; Su, H.; Aili, D. Sci. Rep. 2015, 5, 14063. 